\title{
Simplified Homogeneous Balance Method and Its Applications to the Whitham-Broer-Kaup Model Equations
}

\author{
Mingliang Wang1,2, Xiangzheng Li ${ }^{1}$ \\ ${ }^{1}$ School of Mathematics \& Statistics, Henan University of Science \& Technology, Luoyang, China \\ ${ }^{2}$ Department of Mathematics, Lanzhou University, Lanzhou, China \\ Email: mlwang@haust.edu.cn
}

Received 5 June 2014; revised 3 July 2014; accepted 11 July 2014

Copyright @ 2014 by authors and Scientific Research Publishing Inc.

This work is licensed under the Creative Commons Attribution International License (CC BY).

http://creativecommons.org/licenses/by/4.0/

c) (i) Open Access

\section{Abstract}

A nonlinear transformation of the Whitham-Broer-Kaup (WBK) model equations in the shallow water small-amplitude regime is derived by using a simplified homogeneous balance method. The WBK model equations are linearized under the nonlinear transformation. Various exact solutions of the WBK model equations are obtained via the nonlinear transformation with the aid of solutions for the linear equation.

\section{Keywords}

WBK Model Equations, Simplified Homogeneous Balance Method, Nonlinear Transformation, Multiple Soliton Solutions, Periodic Solutions in Space Variable, Rational Solutions

\section{Introduction}

The Whitham-Broer-Kaup model equations (WBK) [1]-[5] in the shallow water small-amplitude regime are that

$$
\begin{gathered}
u_{t}+u u_{x}+v_{x}+\beta u_{x x}=0, \\
v_{t}+(u v)_{x}-\beta v_{x x}+\alpha u_{x x x}=0,
\end{gathered}
$$

where $u=u(x, t)$ represents the horizontal velocity, and $v=v(x, t)$ the height deviated from the equilibrium position of the liquid, $\alpha$ and $\beta$ are constants. The WBK models (1) and (2) are very good models to describe dispersive waves. If $\alpha=0, \beta \neq 0$, Equations (1) and (2) describe a shallow water waves with diffusion; if $\alpha=1$, $\beta=0$, Equations (1) and (2) become the variant Boussinesq equations. In the latest paper [6], the multiple soliton solutions of Equations (1) and (2) have been obtained by using the simplified form of Hirota's direct method. 
In the present paper, we will apply a simplified homogeneous balance method to investigate the WBK model Equations (1) and (2), by this method a nonlinear transformation that from the solution for a linear equation to the solution for the WBK model equations is derived, and more type of solutions than those given in [6] are obtained via the nonlinear transformation successfully.

\section{Derivation of the Nonlinear Transformation}

Considering the homogeneous balance between $u u_{x}$ and $u_{x x}$ in Equation (1), and between $(u v)_{x}$ and $u_{x x x}$ in Equation (2) $(2 m+1=m+2, m+n+1=m+3$, which implies that $m=1, n=2)$, we can suppose that the solution of Equations (1) and (2) is of the form

$$
u=A(\ln \phi)_{x}, \quad v=B(\ln \phi)_{x x},
$$

where we use $A \ln \phi$ and $B \ln \phi$ instead of the undetermined functions $f(\phi)$ and $g(\phi)$ appearing in the original homogeneous balance method (HB) [7]-[9] to simplify the original HB, constants $A$ and $B$, and the function $\phi=\phi(x, t)$ are to be determined later. The aim of the simplified HB is to find $A$ and $B$, and the function $\phi=\phi(x, t)$ such that the expressions (3) exactly satisfies Equations (1) and (2).

Substituting (3) into the left hand sides of Equations (1) and (2), yields

$$
\begin{gathered}
u_{t}+u u_{x}+v_{x}+\beta u_{x x}=A\left[\frac{\phi_{t}+\left(\frac{B}{A}+\beta\right) \phi_{x x}}{\phi}+\frac{\left(\frac{1}{2} A-\frac{B}{A}-\beta\right) \phi_{x}^{2}}{\phi^{2}}\right]_{x} \\
v_{t}+(u v)_{x}-\beta v_{x x}+\alpha u_{x x x}=B\left[\frac{\phi_{t}+\left(\frac{\alpha A}{B}-\beta\right) \phi_{x x}}{\phi}+\frac{\left(\frac{1}{2} A-\frac{\alpha A}{B}+\beta\right) \phi_{x}^{2}}{\phi^{2}}\right]
\end{gathered}
$$

In order to determine $A$ and $B$, we set the coefficients of the terms with $\frac{\phi_{x}^{2}}{\phi^{2}}$ appearing in expressions (4) to zero, yields algebraic equations for $A$ and $B$

$$
\frac{1}{2} A-\frac{B}{A}-\beta=0, \quad \frac{1}{2} A-\frac{\alpha A}{B}+\beta=0 .
$$

Solving the algebraic equations we have

$$
A= \pm 2 \sqrt{\alpha+\beta^{2}}, \quad B=2\left(\alpha+\beta^{2} \mp \beta \sqrt{\alpha+\beta^{2}}\right) .
$$

Substituting (6) into (3), yields

$$
u= \pm 2 \sqrt{\alpha+\beta^{2}}(\ln \phi)_{x}, \quad v=2\left(\alpha+\beta^{2} \mp \beta \sqrt{\alpha+\beta^{2}}\right)(\ln \phi)_{x x} .
$$

Using (5) and (6), the expressions (4) become

$$
\begin{gathered}
u_{t}+u u_{x}+v_{x}+\beta u_{x x}= \pm 2 \sqrt{\alpha+\beta^{2}}\left[\frac{\phi_{t} \pm \sqrt{\alpha+\beta^{2}} \phi_{x x}}{\phi}\right]_{x}=0, \\
v_{t}+(u v)_{x}-\beta v_{x x}+\alpha u_{x x x}=2\left(\alpha+\beta^{2} \mp \beta \sqrt{\alpha+\beta^{2}}\right)\left[\frac{\phi_{t} \pm \sqrt{\alpha+\beta^{2}} \phi_{x x}}{\phi}\right]_{x x}=0,
\end{gathered}
$$

provided that the function $\phi=\phi(x, t)$ satisfies the linear equation

$$
\phi_{t} \pm \sqrt{\alpha+\beta^{2}} \phi_{x x}=0 .
$$

Based upon (7), (8) and (9), we come to the conclusion that inserting each solution of the linear equation (9) into (7), we can obtain the exact solution of the WBK model Equations (1) and (2), and the expressions (7) with linear Equation (9) can be looked upon as a nonlinear transformation that from the solution for linear Equation (9) to the solution for WBK model Equations (1) and (2), because every solution of linear Equation (9) under (7) 
is transformed into the solution of the WBK model Equations (1) and (2), therefore the WBK model Equations (1) and (2) can be linearized by the linear Equation (9), according to [10], the WBK model equations are C-integrable equations.

\section{Exact Solutions of the WBK Model Equations}

According to the superposition principle for a linear problem, the linear Equation (9) can admit many solutions, for example,

$$
\begin{gathered}
\phi_{1}=1+\sum_{i=1}^{N} c_{i} \mathrm{e}^{\eta_{i}}, \quad \eta_{i}=k_{i} x \mp k_{i}^{2} t, i=1,2, \cdots, N . \\
\phi_{2}=1+\sum_{i=1}^{N} c_{i} \mathrm{e}^{ \pm \omega_{i} t} \cos k_{i} x, \quad \omega_{i}=k_{i}^{2} \sqrt{\alpha+\beta^{2}}, \\
\phi_{3}=1+\sum_{i=1}^{N} c_{i} \mathrm{e}^{ \pm \omega_{i} t} \sin k_{i} x, \quad \omega_{i}=k_{i}^{2} \sqrt{\alpha+\beta^{2}}, \\
\phi_{4}=x^{3}+x^{2}+x \mp(6 x+2) \sqrt{\alpha+\beta^{2}} t+1,
\end{gathered}
$$

and so on., where integer $N \geq 1, k_{i}, c_{i}(i=1,2, \cdots, N)$ are constants.

Substituting (10) into (7), we have the multiple soliton solutions of the WBK model Equations (1) and (2) as follows

$$
\begin{gathered}
u_{1}= \pm 2 \sqrt{\alpha+\beta^{2}} \frac{\sum_{i=1}^{N} c_{i} k_{i} \mathrm{e}^{\eta_{i}}}{1+\sum_{i=1}^{N} c_{i} \mathrm{e}^{\eta_{i}}}, \quad \eta_{i}=k_{i} x \mp k_{i}^{2} t, i=1,2, \cdots, N \\
v_{1}=2\left(\alpha+\beta^{2} \mp \beta \sqrt{\alpha+\beta^{2}}\right)\left[\frac{\sum_{i=1}^{N} c_{i} k_{i}^{2} \mathrm{e}^{\eta_{i}}}{1+\sum_{i=1}^{N} c_{i} \mathrm{e}^{\eta_{i}}}-\frac{\left(\sum_{i=1}^{N} c_{i} k_{i} \mathrm{e}^{\eta_{i}}\right)^{2}}{\left(1+\sum_{i=1}^{N} c_{i} \mathrm{e}^{\eta_{i}}\right)^{2}}\right] .
\end{gathered}
$$

If $N=1,2,3$ the expressions (14) become the 1-soliton solutions, 2-solliton solutions and 3-soliton solutions for the WBK model equations, respectively, these results coincide with those obtained by using the simplified form of Hirota's method in [6] one by one. In particular, when $N=1, c_{1}=1, k_{1}=k$, (14) becomes

$$
\begin{gathered}
u= \pm k \sqrt{\alpha+\beta^{2}}\left[1+\tanh \frac{1}{2}\left(k x \mp k^{2} \sqrt{\alpha+\beta^{2}} t\right)\right], \\
v=\frac{1}{2}\left(\alpha+\beta^{2} \mp \beta \sqrt{\alpha+\beta^{2}}\right) k^{2} \operatorname{sech}^{2} \frac{1}{2}\left(k x \mp k^{2} \sqrt{\alpha+\beta^{2}} t\right),
\end{gathered}
$$

where $u$ represents a single kink solitary wave, and $v$ a single bell solitary wave of the WBK model Equations (1) and (2).

Substituting (11) into (7), we have the periodic solutions in space variable $x$ for the WBK model Equations (1) and (2)

$$
\begin{gathered}
u_{2}=\mp 2 \sqrt{\alpha+\beta^{2}} \frac{\sum_{i=1}^{N} c_{i} k_{i} \mathrm{e}^{ \pm \omega_{i} t} \sin k x_{i}}{1+\sum_{i=1}^{N} c_{i} \mathrm{e}^{ \pm \omega_{i} t} \cos k_{i} x}, \quad \omega_{i}=k_{i}^{2} \sqrt{\alpha+\beta^{2}}, \\
v_{2}=-2\left(\alpha+\beta^{2} \mp \beta \sqrt{\alpha+\beta^{2}}\right)\left[\frac{\sum_{i=1}^{N} c_{i} k_{i}^{2} \mathrm{e}^{ \pm \omega_{i} t} \cos k_{i} x}{1+\sum_{i=1}^{N} c_{i} \mathrm{e}^{ \pm \omega_{i} t} \cos k_{i} x}+\frac{\left(\sum_{i=1}^{N} c_{i} k_{i} \mathrm{e}^{ \pm \omega t_{i}} \sin k_{i} x\right)^{2}}{\left(1+\sum_{i=1}^{N} c_{i} \mathrm{e}^{ \pm \omega_{i} t} \cos k_{i} x\right)^{2}}\right] .
\end{gathered}
$$


Similarly, substituting (12) into (7), we also have the periodic solutions in space variable $x$ for the WBK model Equations (1) and (2)

$$
\begin{gathered}
u_{3}= \pm 2 \sqrt{\alpha+\beta^{2}} \frac{\sum_{i=1}^{N} c_{i} k_{i} \mathrm{e}^{ \pm \omega_{i} t} \cos k x_{i}}{1+\sum_{i=1}^{N} c_{i} \mathrm{e}^{ \pm \omega_{i} t} \sin k_{i} x}, \quad \omega_{i}=k_{i}^{2} \sqrt{\alpha+\beta^{2}}, \\
v_{3}=-2\left(\alpha+\beta^{2} \mp \beta \sqrt{\alpha+\beta^{2}}\right)\left[\frac{\sum_{i=1}^{N} c_{i} k_{i}^{2} \mathrm{e}^{ \pm \omega_{i} t} \sin k_{i} x}{1+\sum_{i=1}^{N} c_{i} \mathrm{e}^{ \pm \omega_{i} t} \sin k_{i} x}-\frac{\left(\sum_{i=1}^{N} c_{i} k_{i} \mathrm{e}^{ \pm \omega_{i} t} \cos k_{i} x\right)^{2}}{\left.\left(1+\sum_{i=1}^{N} c_{i} \mathrm{e}^{ \pm \omega_{i} t} \sin k_{i} x\right)^{2}\right] .}\right.
\end{gathered}
$$

Substituting (13) into (7), we have rational solutions for the WBK model Equations (1) and (2)

$$
\begin{gathered}
u_{4}=2 \sqrt{\alpha+\beta^{2}} \frac{3 x^{2}+2 x+1 \mp 6 \sqrt{\alpha+\beta^{2}} t}{x^{3}+x^{2}+x \mp(6 x+2) \sqrt{\alpha+\beta^{2}} t+1}, \\
v_{4}=2\left(\alpha+\beta^{2} \mp \beta \sqrt{\alpha+\beta^{2}}\right)\left\{\frac{\left(3 x^{2}+2 x \mp 6 \sqrt{\alpha+\beta^{2}} t\right)^{2}}{x^{3}+x^{2}+x \mp(6 x+2) \sqrt{\alpha+\beta^{2}} t+1}-\frac{6 x+2}{\left[x^{3}+x^{2}+x \mp(6 x+2) \sqrt{\alpha+\beta^{2}} t+1\right]^{2}}\right\}
\end{gathered}
$$
[6].

We point out that the solutions $\left(u_{2}, v_{2}\right),\left(u_{3}, v_{3}\right),\left(u_{4}, v_{4}\right)$ to the WBK model equations have not appeared in

\section{Conclusion}

In this paper, the original HB is simplified by using a logarithmic function instead of the undetermined function appearing in the original HB. The nonlinear transformation that from the solution for the linear equation to the solution for the WBK model equation is derived by using the simplified HB. The WBK model equations are linearized under the nonlinear transformation. The multiple soliton solutions, periodic solutions in space variable and rational solutions of the WBK model equations are obtained in terms of solutions for the linear equation.

\section{Acknowledgements}

This work is supported in part by the Natural Science Foundation of Education Department of Henan Province of China (Grant No. 2011B110013, 12B110006) and the Doctoral Foundation of Henan University of Science and Technology (Grant No. 09001562).

\section{References}

[1] Whitham, G.B. (1967) Variational Methods and Applications to Water Waves. Proceedings of the Royal Society A, 299, 6-25. http://dx.doi.org/10.1098/rspa.1967.0119

[2] Broer, L.J. (1975) Approximate Equations for Long Water Waves. Applied Scientific Research, 31, 377-395. http://dx.doi.org/10.1007/BF00418048

[3] Kaup, D.J. (1975) A Higher-Order Water-Wave Equation and the Method for Solving It. Progress of Theoretical Physics, 54, 396-408. http://dx.doi.org/10.1143/PTP.54.396

[4] Kupershmidt, B.A. (1985) Mathematics of Dispersive Waves. Communications in Mathematical Physics, 99, 51-73. http://dx.doi.org/10.1007/BF01466593

[5] Fan, E. and Zhang, H. (1998) Backlund Transformation and Exact Solutions for Whitham-Broer-Kaup Equations in Shallow Water. Applied Mathematics and Mechanics, 19, 713-716. http://dx.doi.org/10.1007/BF02457745

[6] Wazwaz, A.M. (2013) Multiple Soliton Solutions for the Whitham-Broer-Kaup Model in Shallow Water Small-Amplitude Regime. Physica Scripta, 88. http://dx.doi.org/10.1088/0031-8949/88/03/035007

[7] Wang, M.L. (1995) Solitary Wave Solutions for Variant Boussinesq Equations. Physics Letters A, 199, 169-172. http://dx.doi.org/10.1016/0375-9601(95)00092-H 
[8] Wang, M.L. (1996) Exact Solutions for a Compound KdV-Burgers Equation. Physics Letters A, 213, 279-287. http://dx.doi.org/10.1016/0375-9601(96)00103-X

[9] Wang , M.L., Zhou, Y.B. and Li, Z.B. (1996) Application of a Homogeneous Balance Method to Exact Solutions of Nonlinear Equations in Mathematical Physics. Physics Letters A, 216, 67-75.

http://dx.doi.org/10.1016/0375-9601(96)00283-6

[10] Calogero, F. (1991) Springer Series in Nonlinear Dynamics. Zakharov, V.E., Ed., Springer, Berlin, 1-62. 
Scientific Research Publishing (SCIRP) is one of the largest Open Access journal publishers. It is currently publishing more than 200 open access, online, peer-reviewed journals covering a wide range of academic disciplines. SCIRP serves the worldwide academic communities and contributes to the progress and application of science with its publication.

Other selected journals from SCIRP are listed as below. Submit your manuscript to us via either submit@scirp.org or Online Submission Portal.
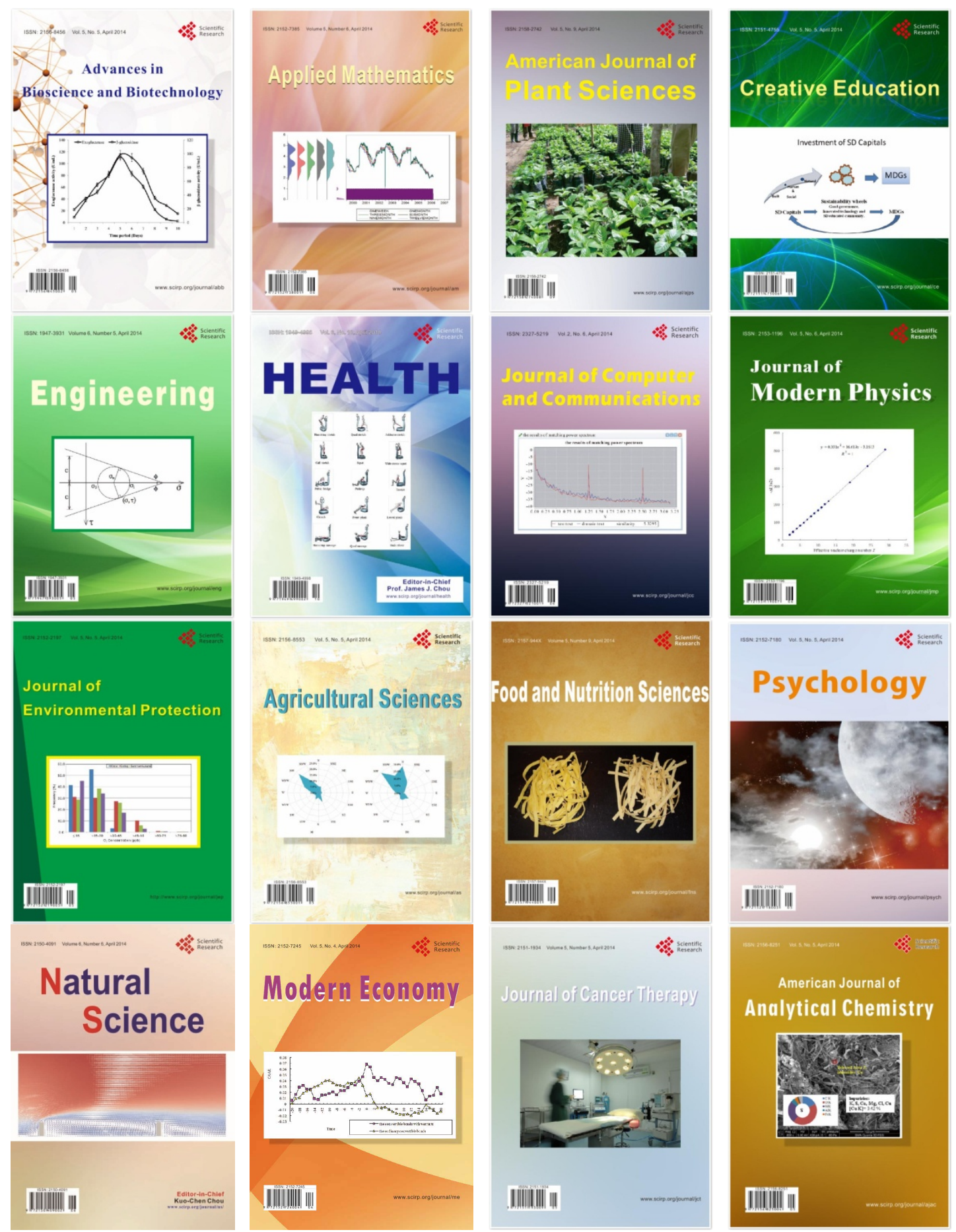